\title{
Intractable Desquamative Interstitial Pneumonia in a Tattooed Man
}

\author{
Toru Arai ${ }^{1}$, Yoshikazu Inoue ${ }^{1}$, Seiji Hayashi ${ }^{1}$, Masanori Akira ${ }^{1}$, Satoru Yamamoto ${ }^{1}$, \\ William D Travis ${ }^{2}$ and Mitsunori Sakatani ${ }^{1}$
}

\begin{abstract}
A 20-year-old man with a 15 pack-year history of cigarette smoking had a tattoo outlined on his back with blue pigment. He noticed a dry cough and shortness of breath on exertion when the pigment of other colors was added at the age of 27. He visited our hospital two years later because of severe dyspnea. He was diagnosed with desquamative interstitial pneumonia by surgical lung biopsy. Steroid therapy with cessation of smoking was partially effective, however his disease worsened again and he died three and a half years after the diagnosis because of respiratory failure.
\end{abstract}

Key words: desquamative interstitial pneumonia, tattoo, foreign body, respiratory failure

(DOI: 10.2169/internalmedicine.45.1727)

\section{Introduction}

Desquamative interstitial pneumonia (DIP) is histologically characterized by intraalveolar accumulation of macrophages and was first described by Liebow in 1965 (1). The etiology of this disease is unknown, but more than $90 \%$ of patients with DIP are smokers and have the potential for DIP to resolve spontaneously with cessation of smoking. Carrington et al (2) reported that patients with DIP show a better prognosis and response to steroid therapy than those with usual interstitial pneumonia (2-4). We report herein a patient with DIP, who developed shortness of breath simultaneously with tattooing and died from respiratory failure despite therapy with steroids as well as smoking cessation.

\section{Case Report}

A 20-year -old male clerical employee without dust exposure and no pets had his back tattooed using blue pigment. He noticed a dry cough and shortness of breath on exertion at the age of 27 when the pigment of other colors was started to be utilized for his tattoo. Dyspnea on exertion progressively worsened over about two years and he presented at our hospital at the age of 29. Although he had smoked one pack of cigarettes daily for 15 years, he stopped smoking because of dyspnea. Neither inspiratory crackles, digital clubbing nor cutaneous lesions were revealed by physical examinations. Pulmonary function tests demonstrated severe restrictive pattern with vital capacity of $1.18 \mathrm{~L}(27.9 \%$ predicted), and diffusing capacity of the lung for carbon monoxide was $18.5 \%$ of predicted. The findings of arterial blood gas analysis under 2 liters of oxygen supply were $\mathrm{pH} 7.413$, $\mathrm{PaCO}_{2}$ 41.1 Torr, $\mathrm{PaO}_{2}$ 63.3 Torr. His chest radiograph showed bilateral infiltrative shadows predominantly in the lower lung field and suggested loss of lung volume (Fig. 1). High-resolution computed tomography (HRCT) provided ground glass opacity (GGO) and traction bronchiectasis predominantly in bilateral lower lobes (Fig. 2). Laboratory examination showed white blood cells 8,700/ $\mu \mathrm{l}$, C-reactive protein $0.30 \mathrm{mg} / \mathrm{dl}$ and elevation of lactate dehydrogenase to 534 IU/L. Peripheral blood eosinophilia was not observed throughout his clinical course.

Bronchoalveolar lavage fluid (BALF) was obtained from the left $\mathrm{B}^{4}$ bronchus with $150 \mathrm{~mL}$ of saline and $23 \%$ of the fluid was recovered. Total cell count in BALF was $3.04 \times$ $10^{5} / \mathrm{mL}$ and the differentiation of the cells was macrophages $34.3 \%$, neutrophils $17.5 \%$, lymphocytes $3.1 \%$ and eosino-

\footnotetext{
${ }^{1}$ National Hospital Organization Kinki-chuo Chest Medical Center, Osaka and ${ }^{2}$ Department of Pathology, Memorial Sloan Kettering Cancer Center, New York, NY, U.S.A.

Received for publication January 10, 2006; Accepted for publication June 12, 2006

Correspondence to Dr. Yoshikazu Inoue, National Hospital Organization Kinki-chuo Chest Medical Center, 1180 Nagasone-cho, Kita-ku, Sakai, Osaka 591-8555
} 


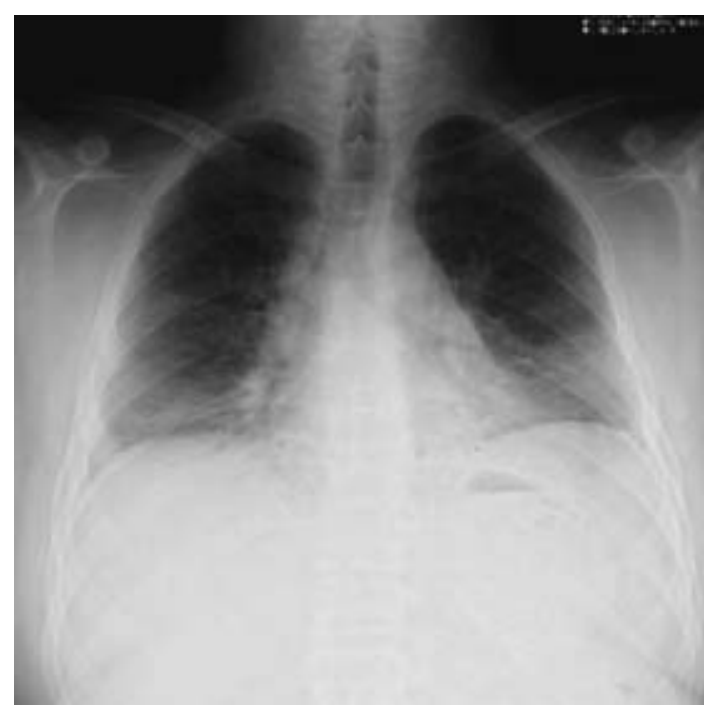

Figure 1. Chest radiograph on the first admission showed bilateral infiltrative shadows predominantly in lower lung field and suggested loss of lung volume.

phils $45.1 \%$. The CD4+/CD8+ ratio of lymphocytes in BAL, determined by flow cytometry, was 0.79 . Transbronchial lung biopsy proved to be nondiagnostic. The lung specimens obtained by video-assisted thoracoscopic surgery (VATS) showed temporal uniformity. Alveolar walls were thickened by lymphocytes and plasma cells, and alveolar spaces were filled with large numbers of pigmented macrophages, but with scant eosinophils, which were compatible with the DIP pattern (Fig. 3) (3). Findings of his HRCT and BALF were consistent with DIP $(5,6)$.

He underwent high-dose intravenous methylprednisolone therapy of $1 \mathrm{~g}$ daily for three days and after that $60 \mathrm{mg}$ of oral prednisolone daily was started and reduced to $20 \mathrm{mg}$ daily after 4 months. His cough and shortness of breath were resolved and findings of arterial blood gas analysis on room air improved to $\mathrm{pH} 7.405, \mathrm{PaCO}_{2} 43.3$ Torr, $\mathrm{PaO}_{2} 89.6$ Torr. After that he experienced four relapses of his lung disease in spite of smoking cessation and immunosuppressive therapy. He died from respiratory failure three and a half years after the diagnosis.

\section{Discussion}

Patients with DIP usually respond to steroid therapy and demonstrate a better prognosis than idiopathic pulmonary fibrosis (2-4). Although the patho-physiology of DIP is not precisely elucidated, it is suggested by many authors that DIP is related to smoking and spontaneous resolution is possible after smoking cessation $(3,7)$. However, unknown etiologies other than smoking are potential causes of DIP in some cases, and they often demonstrated a poor response to steroid and have an unfavorable prognosis $(8,9)$.

The relationship between DIP and a systemic disease has been suggested in some cases. King et al reported a female patient with progressive DIP (10). Although she discontin- ued smoking and was treated with steroid and cyclophosphamide, she became worse and received lung transplantation three years after diagnosis. Her disease recurred in the allograft and she died eight months after the transplant. Genetic factors are thought to be associated with some cases of DIP. Childhood cases of DIP $(11,12)$ often happened familiarly in patients under the age of 6 months old and the patients died in about three years despite the use of steroids and other immunosuppressive agents. Recently, it was shown that mutations in the surfactant protein-C (SP-C) gene are associated with familial DIP $(13,14)$ and the mutations are found in the adult cases $(13,14)$. Thus, we should keep in mind various factors possibly associated with DIP.

Cessation of smoking was ineffective in the present case and his unfavorable prognosis suggests an association with an unknown etiology other than smoking. It is difficult to prove that the cessation of the patient's smoking has been maintained. However, his lung disease did not improve spontaneously although he could not smoke in our hospital. No genetic background was indicated from his family history, or mutations of SP-C gene were not investigated. Infection was ruled out by examination of his surgical lung biopsy specimens and serological findings. If we consider something other than smoking may have caused his poor prognostic disease, we suppose a possibility is tattooing, because his cough and dyspnea occurred soon after he had his back tattooed with several pigments.

Pulmonary disease can be induced as a result of reaction to a foreign body outside the lungs. It is well known that silicone used for cosmetic breast-enlargement surgery can induce autoimmune diseases (15) and interstitial pneumonia (16). Tattooing can also arouse a foreign body reaction. The occurrence of granulomas in a tattoo is a well-known phenomenon $(17,18)$, and systemic granulomatous disease like sarcoidosis with uveitis and pulmonary lesions is also reported (19). Defects of local skin lesions cannot disallow a relationship between the tattoo and DIP in the present case, because pulmonary granulomas, associated with tattooing without remarkable skin lesion, is reported (20) and a silicone implant generally shows no local reaction in the breast.

We should also suppose the possibility of other diseases with increased eosinophils in BAL fluid. Chronic eosinophilic pneumonia (CEP) was characterized by intra-alveolar eosinophils, macrophages and amorphous proteinous exudates and CEP resembles DIP, especially in patients who have received steroids prior to the lung biopsy (21). However, eosinophil infiltration was not prominent in the VATS specimens of this case before the treatment. BAL eosinophilia has also been reported in DIP cases $(22,23)$. Thus, we suppose the histologic findings of this case are consistent with DIP rather than CEP, and he was finally diagnosed with DIP after our clinical, radiological and pathological conference.

Investigation of additional cases in the future may be necessary to make any more definite conclusions about the relationship, however, it is important to describe the possible 


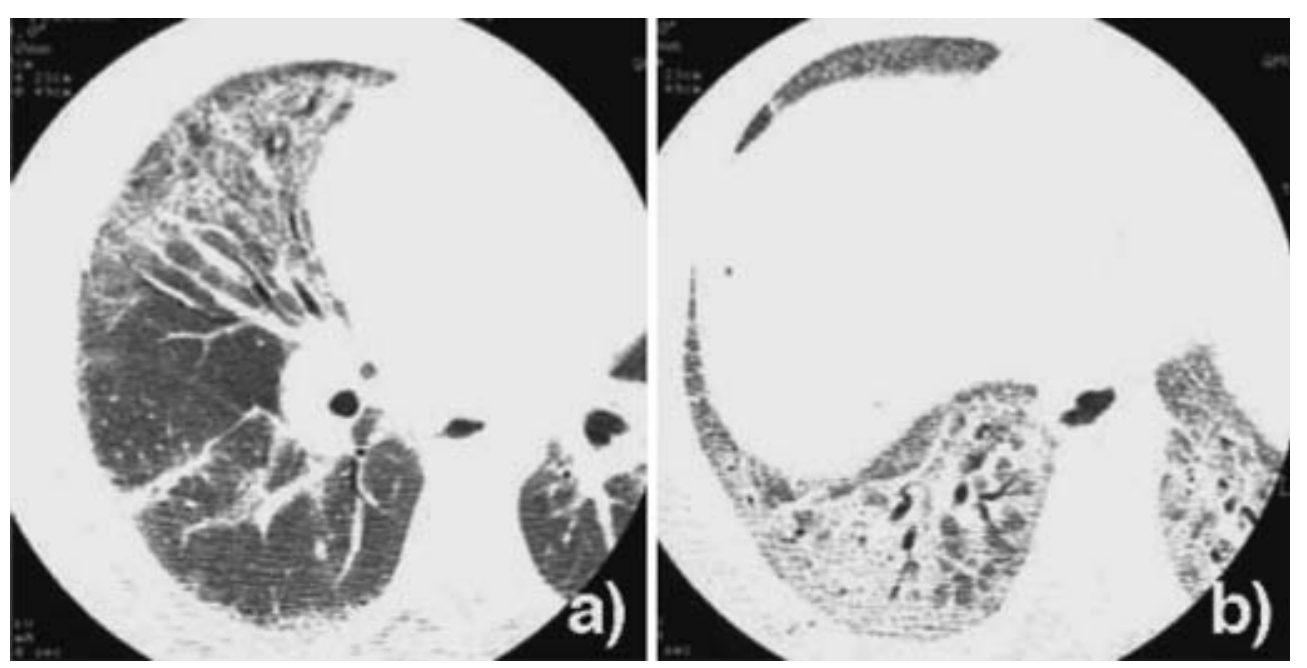

Figure 2. High-resolution computed tomography on the first admission. The ground glass opacity (GGO) was shown in the middle lobe (Fig. 2a) and GGO and traction bronchiectasis predominantly in the lower lobe (Fig. 2b).

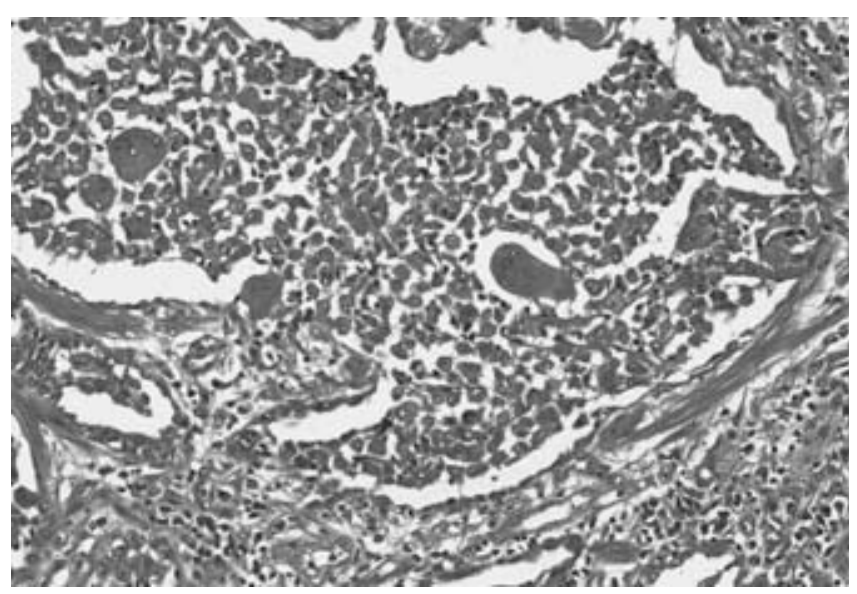

Figure 3. Microscopic findings of lung specimens obtained by video-assisted thoracoscopic surgery. HE staining. Alveolar walls were thickened with lymphocytes and plasma cells and alveoli were filled with pigmented alveolar macrophages. These findings were compatible with a desquamative interstitial pneumonia pattern. cause of intractable cases of DIP.

Diagnosis of this case was discussed and ascertained in the Osaka Meeting on Diffuse Infiltrative Lung Diseases (August 28, 1999). We thank the members of the meeting for their useful discussion.

\section{References}

1. Liebow AA, Steer A, Billingsley JG. Desquamative interstitial pneumonia. Am J Med 39: 369-404, 1965.

2. Carrington CB, Ceansler EA, Coutou RE, FitzGerald MX, Gupta RG. Natural history of treated course of usual and desquamative interstitial pneumonia. N Engl J Med 298: 801-809, 1978.

3. American Thoracic Society. European Respiratory Society. International multidisciplinary consensus classification of the idiopathic interstitial pneumonias. Am J Respir Crit Care Med 165: 277304, 2002.

4. Travis WD, Matsui K, Moss J, Ferrans VJ. Idiopathic nonspecific interstitial pneumonia: Prognostic significance of cellular and fibrosing patterns. Survival comparison with usual interstitial pneumonia and desquamative interstitial pneumonia. Am J Surg Pathol 24: 19-33, 2000.
5. Zompatori M, Bna C, Poletti V, et al. Diagnostic imaging of diffuse infiltrative disease of the lung. Respiration 71: 4-19, 2004.

6. Poletti V, Chilosi M, Olivieri D. Diagnostic invasive procedures in diffuse infiltrative lung diseases. Respiration 71: 107-119, 2004.

7. Matsuo K, Tada S, Kataoka M, Okahara M, et al. Spontaneous remission of desquamative interstitial pneumonia. Intern Med 36: 728-731, 1997.

8. Flusser G, Gurman G, Zirkin H, Prinslo I, Heimer D. Desquamative interstitial pneumonits causing acute respiratory failure, responsive only to immunosuppressants. Respiration 58: 324-326, 1991.

9. Gould TH, Buist MD, Meredith D, Thomas PD. Fulminant desquamative interstitial pneumonitis. Anaesth Intensive Care 26: 677-679, 1998. 
10. King MB, Jessurun J, Hertz MI. Recurrence of desquamative interstitial pneumonia after lung transplantation. Am J Respir Crit Care Med 156: 2003-2005, 1997.

11. Buchino JJ, Keenan WJ, Algren JT, Bove KE. Familial desquamative interstitial pneumonitis occurring in infants. Am J Med Genet Suppl 3: 285-291, 1987.

12. Tsukahara M, Yoshii H, Imamura $T$, Kamei T, Koga M, Furukawa S. Desquamative interstitial pneumonia in sibs. Am J Med Genet 59: 431-434, 1995 .

13. Nogee LM, Dunber AE 3rd, Wert SE, Askin F, Hamvas A, Whitsett JA. A mutation in surfactant protein $\mathrm{C}$ gene associated with familial interstitial lung disease. N Engl J Med 344: 573-579, 2001.

14. Thomas AQ, Lane K, Phillips J 3rd, et al. Heterozygosity for a surfactant protein $\mathrm{C}$ gene mutation associated with usual interstitial pneumonitis and cellular nonspecific interstitial pneumonitis in one kindred. Am J Respir Crit Care Med 165: 1322-1328, 2002.

15. Spiera RF, Gibofsky A, Spiera H. Silicone gel filled breast implants and connective tissue disease: an overview. J Rheumatol 21 239-245, 1994

16. Lai YF, Chao TY, Wong SL. Acute pneumonitis after subcutaneous injections of silicone for augmentation mammaplasty. Chest
106: 1152-1155, 1994.

17. Obermayer ME, Hassen M. Sarcoidosis with sarcoidal reaction in tattoo. Arch Dermatol 71: 766, 1955.

18. Collins P, Evans AT, Gray W, Levison DA. Pulmonary sarcoidosis presenting as a granulomatous tattoo reaction. Br J Dermatol 130: 658-662, 1994.

19. Rorsman H, Brehmer-Andersson E, Dahliquist I, Jacobsson S, Linell F, Rorsman G. Tattoo granuloma and uveitis. Lancet 2: 2728, 1969.

20. Colp CR, Goldfarb R, Ongseng F. Value of gallium imaging in the evaluation of tattoo granulomas due to sarcoidosis. Chest 100: 1737-1738, 1991.

21. Travis WD, Colby TV, Koss MN. Non-neoplastic Disorders of the Lower Respiratory Tract. American Registry of Pathology, Washington, 2002: 160-169.

22. Veeraraghavan S, Latsi PI, Wells AU, et al. BAL findings in idiopathic nonspecific interstitial pneumonia and usual interstitial pneumonia. Eur Respir J 22: 239-244, 2003.

23. Sato N, Kawabata Y, Takayanagi N, et al. A case of desquamative interstitial pneumonia with bilateral hilar and mediastinal lymphadenopathy. Nihon Kokyuki Gakkai Zasshi 42: 446-453, 2004.

(C) 2006 The Japanese Society of Internal Medicine http://www.naika.or.jp/imindex.html 